\title{
Reflections on a Patient Suicide in the Time of COVID-19
}

\author{
Yi Yen Annie Yeh ${ }^{1}$
}

Received: 12 May 2020 / Accepted: 15 October 2020 /Published online: 23 October 2020

(C) Academic Psychiatry 2020

I lost one of my patients to suicide in my second year of residency. This experience was devastating. I felt helpless, defeated, and debilitated by self-doubt. "Hadn't I gone into medicine to save lives?" No amount of reassurance by my mentors could assuage the guilt and grief I felt for the death of an 18-year-old patient who decided to end his life. I had no playbook for how to talk to his family nor how to unpack the overwhelming intensity of emotions I felt. I found myself obsessing over what I could have said or done differently to prevent it. Looking back, I still do not know what, if anything, I would have changed about my encounter and clinical decision making.

A patient's suicide leaves us with more questions than answers. In my case, I questioned the meaning of life and the meaning of my work. Somehow, death by suicide seems worse than other deaths. This incident occurred almost 3 years ago, yet its impact is omnipresent in my interactions with patients, families, and the lessons I teach other residents. I sought out and have continued with my own psychotherapy as a result which has helped me contextualize and integrate this intense experience into a narrative. This has ultimately reinforced for me the reasons why I went into psychiatry. Over time, I have found solace in seeking out conversations with colleagues who have also experienced a patient death by suicide. I chose to give a grand rounds presentation on the topic of patient suicide during residency, exploring how we might support trainees who experience this awful event during a formative time in their professional and personal growth. Serendipitously, I discovered a resident trainee group at the American Psychiatric Association's annual meeting whose purpose is to share and discuss this very important topic. I am incredibly grateful for the mentorship and safe space that belonging to this group has given me to further process my experience of a patient's suicide during training.

Yi Yen Annie Yeh

yyeh1@tulane.edu

1 Tulane University School of Medicine, New Orleans, LA, USA
When COVID-19 arrived in New Orleans in the weeks following Mardi Gras, the anxiety and panic were palpable in my residency program as we prepared for a possible reallocation of residents to ICU and medicine units. In the midst of this uncertainty, a partnership was born out of our psychiatry residency program and the palliative care team at University Medical Center, the current namesake of old Charity hospital, a safety net academic center and one of our training sites. Upper level psychiatry residents engaged in virtual rounds with the interdisciplinary palliative care team every morning and were given assignments to contact families of COVID-19 positive patients in the ICU with updates. For the 3 weeks I was on this service, I became part of a beloved 69-year-old sociology professor's family, talking with one of her adult sons near daily and part of a 35-year-old man's extended family, painfully cognizant of the strained family dynamics between his mother and the grandmother who raised him. My position was unique as a mediator between family members who so desperately wanted to be at their loved ones' bedside and the primary teams who were stretched beyond capacity. In making the time to provide daily updates to families, I was also giving them the space to share with me intimate stories that brought their loved ones to lifestories I relayed back to our team. This window into the lives of my patients who were intubated and unable to communicate their wishes or desires added depth and dimension to their physical bodies.

As we prepared for end-of-life discussions, this storytelling served an important purpose in allowing families to celebrate the lives of their loved ones and healthcare workers to honor our patients' lives in their final days. Through these conversations, families gave us glimpses into our patient's liveswhat they valued, what gave their life meaning. Though difficult, there was a sense of closure in discussing these matters. I often thought about my 18-year-old patient as we navigated these end-of-life discussions. He - unlike my ICU patientswas awake, alert, and could communicate his wishes. Was his true wish when he decided to take his life to die and leave the world forever? I will never know the answer and this question will continue to haunt me. 
We live in a culture that has a tough time talking about death and dying. I truly believe that in our field of medicine, we must talk about this topic, beginning with exploring our own thoughts on death and dying. It is a difficult conversation, no doubt, but one that is paramount to our role as physicians and one that I believe can provide meaning to our everyday lived lives and possibly to those of our patients. As psychiatrists, we have the skills to interview compassionately, listen empathically, and create a safe holding space for patients and families in times of struggle. The words of Dr. Viktor Frankl echo in my mind as I wonder what would have come up for this young man had we had the time to explore the purpose and meaning in his life. He was so young. He did not get to go to collegesomething he had been looking forward to. He had suffered from depression and trauma at a young age and this had indelibly affected his outlook on life. Part of my pain in mourning his death was undoubtedly his young age and the promises and hopes I associate with this stage of human development.

While we may not be able to prevent suicides, I do believe that in psychiatry, we can use our skills to engender hope and resiliency in our patient encounters and in so doing, to hopefully impart a sense of value and purpose in the lives of our patients who are questioning the meaning in life. This is even more important in the era of COVID-19 when the practice of social distancing can further exacerbate feelings of loneliness and contribute to, or worsen, preexisting mental health conditions.

Acknowledgments The author would like to acknowledge Dr. Caley McIntyre, Dr. Kristopher Kaliebe, Anna Maria Signorelli, and Dr. Sidney Zisook for reviewing previous versions of this manuscript and for their continued support, guidance, and mentorship.

\section{Compliance with Ethical Standards}

Disclosurest The author states that there is no conflict of interest.

Publisher's Note Springer Nature remains neutral with regard to jurisdictional claims in published maps and institutional affiliations. 